\title{
String Theory on Dp-plane waves
}

\author{
Jose F. Morales \\ Institute for Theoretical Physics and Spinoza Institute, \\ Utrecht University, Postbus 80.195, 3508 TD Utrecht, The Netherlands
}

\begin{abstract}
We study the spectrum of solvable string models on plane waves descending from non-conformal Dp-brane geometries. We mainly focus on S-dual F1/D1waves in type IIB and type I/heterotic 10D superstrings. We derive the Kaluza-Klein spectrum of $\mathcal{N}=1,210 \mathrm{D}$ supergravities on D1/F1-waves. We compute helicity supertraces counting multiplicities and $\mathcal{R}$-charges of string excitations in the plane wave geometry. The results are compared against the expectations coming from gauge/supergravity descriptions. In the type I case, the Klein, Annulus and Moebius one-loop amplitudes are computed for ten-dimensional D1-waves. We test the consistency of the open string descendant by showing that after modular transformations to the closed string channel, the three amplitudes combine themselves to reconstruct a complete square $(|B\rangle+|C\rangle)^{2}$. Tadpole conditions are also discussed.
\end{abstract}

KEYWORDs: ppw ads dbr. 


\section{Contents}

1. Introduction 1

2. Penrose limits of Dp-branes 3

3. KK supergravity harmonics 5

3.1 Type IIB supergravity 7

3.2 Type I supergravity 9

4. String theory on Dp-plane waves 10

4.1 The torus amplitude 11

4.2 Unoriented and open strings 13

5. Summary and open questions 16

\section{Introduction}

According to [1], gauge theories at large $\mathrm{N}$ can often be rephrased in terms of holographically related string theories on $A d S \times S$ spaces pierced by $\mathrm{RR}$ fluxes. The typical example relates $\mathcal{N}=4 \mathrm{SYM}$ to type IIB string theory on $A d S_{5} \times S^{5}$. Superstring theories on such backgrounds are still far from being tractable, obstructing a more quantitative study of the correspondence beyond the classical supergravity level. An unexpected handle for this physics comes from [2], where a solvable string theory was proposed as an effective description of the bulk physics in the nearby of null geodesics of $A d S_{5} \times S^{5}$. The geometry is defined by a Penrose limit along a generic null geodesic of $A d S_{5} \times S^{5}$ and is given in terms of a pp-wave metric supported by a self-dual five-form [3]. In the gauge theory side, the limit corresponds to isolate composite operators with large $\mathcal{R}$-charge.

Plane wave geometries are exact solutions of string theory (see [4] and references therein). Under certain conditions they lead to exactly solvable string models where the spectrum and simple correlation functions can be computed much in the same way as in flat space. We are interested in plane waves arising as Penrose limits of pbrane geometries. The rich isometry group of near horizon p-brane solutions relates most of the choices for null geodesics leaving only few independent possibilities. In case one starts with a pure $A d S \times S$ solution, the two independent possibilities lead to either a pp-wave or a flat space-time [3]. 
In [5, 6], the Penrose limits of non-conformal Dp brane geometries were considered. The limit along a generic null geodesic results again into a pp-wave solution, but now with running dilaton, masses and RR-fluxes. Remarkably, as first noticed in [6], the geometries display a "critical radius" which acts as a fixed point in the radial evolution of a particular class of null geodesics. The physics along such "critical" geodesics is accurately described by a solvable string theory with constant masses, dilaton and RR fluxes and will be the main subject of our study.

Following the BMN ideas, it is natural to consider string theories on these solvable pp-wave vacua as the holographic duals of states with large $\mathcal{R}$-charge in the SYM theories leaving on the branes. The worldvolume theories leaving on Dp-branes are defined via dimensional reduction from $\mathcal{N}=1 \mathrm{SU}(\mathrm{N}) \mathrm{SYM}$ in $D=10$ down to $d=p+1$. For $p \neq 3$, they are non-conformal gauge theories and preserve sixteen supercharges. Before taking the Penrose limit they are described by warped $A d S$ supergravities. Various aspects of these correspondences have been studied in [7] and more recently in $[8$. The Penrose limit, we are considering here, correspond to a very special limit where $\mathcal{R}$-charges in the SYM theory are taken to be large while keeping fixed the working energy scale. This is of course the closest analog of the "conformal" pp-wave versions of the correspondence. The physical meaning of this "critical energy" in the SYM side remains to be fully clarified.

The aim of this paper is to study the spectrum of string theory on general Dpwaves. We would like to stress that solvable Dp-wave string models are interesting by itself. They provide the simplest string vacua with sixteen supercharges and nontrivial RR fluxes. In the absence of a better understanding of string theory on more general RR backgrounds, pp-wave solvable models constitute the unique tractable examples. In the same spirit, string-string dualities in the presence of RR-fields are fully unexplored and Dp plane waves provide a simplified setting where a deeper study of this physics can be addressed.

In this paper we mainly focus on D1-waves in type IIB/type I theory. Besides the duality with 2D SYM theories, these models are interesting because they can be reformulated entirely in terms of NSNS F1-plane waves in the S-dual type IIB/heterotic string respectively. The S-dual pairs are associated to Penrose limits along critical null geodesics of the corresponding p-brane solutions in the low energy supergravities. Type I descendants are constructed by introducing a consistent set of D9-O9 planes in the type IIB D1-wave. It is important to notice that D9-O9 systems are allowed in the D1-wave unlike in the more familiar D3-wave geometry where they were excluded by the selection rules derived in [9, 10, 11].

The paper is organized as follows: In Section 2 we review and adapt the results of [6] in order to derive pp-wave solutions as "critical" Penrose limits of Dp-brane near horizon geometries. Section 3 is devoted to the study of the spectrum of KK harmonics in $\mathcal{N}=1,2$ supergravity on the $\mathrm{D} 1 / \mathrm{F} 1$ plane wave. In section 4 , we compute one-loop modular invariant helicity supertraces counting multiplicities and 
charges of string excitations in the Dp-waves. The formulas encode in a unifying way the resulting spectrum of S-dual pairs of F1/D1 plane waves in 10D superstrings. The Klein, Annulus and Moebius amplitudes defining open descendants of D1-waves are also discussed. In section 5 we summarize the results and comment on interesting directions for future research.

\section{Penrose limits of Dp-branes}

Penrose limits of non-conformal brane solutions have been recently studied in [5, [6]. For $p \neq 3$ and a generic choice of null geodesics the limit results into a pp-wave with $x^{+}$-dependent dilaton, masses and RR-fluxes. However, as noticed in [6], to any

given ratio $\ell \equiv \frac{J}{E}$ of the energy and angular momentum eigenvalues, there exists a critical radius $r_{0}$, in the nearby of which, physics is effectively described by a solvable string theory. We are interested in Penrose limits along geodesics starting near $r_{0}$. In this section we review (and slightly adapt) the results of [5, 6] focusing on this particular class of geodesics. For reasons that will become evident later, we choose to work in the Einstein frame.

Dp-brane solutions involve, besides the metric, non-trivial profiles for the dilaton and $\mathrm{RR}(p+2)$-form. In the Einstein frame the solution can be written as :

$$
\begin{aligned}
d s^{2} & =H^{\frac{p-7}{8}}\left(-d t^{2}+d \vec{x}^{2}\right)+H^{\frac{p+1}{8}}\left(d r^{2}+r^{2} d \psi^{2}+r^{2} \sin ^{2} \psi d \Omega_{7-p}^{2}\right) \\
F_{p+2} & =d \operatorname{vol}\left(\mathbf{E}^{1, p}\right) \wedge d H^{-1} \\
e^{\phi-\phi_{\infty}} & =H^{\frac{3-p}{4}}
\end{aligned}
$$

with $H$ the harmonic function in the transverse space :

$$
H=1+\frac{Q_{p}}{r^{7-p}} \approx \frac{Q_{p}}{r^{7-p}} \quad Q_{p}=c_{p} g_{\mathrm{YM}}^{2} N\left(\alpha^{\prime}\right)^{5-p}
$$

Throughout this paper we restrict ourselves to near horizon regions $r^{7-p} \ll Q_{p}$, where the one in $H$ can be discarded.

We are interested in the physics along the null geodesics:

$$
r=r\left(x^{+}\right) \quad \psi=-b\left(x^{+}\right) \quad t=-\ell \psi-a\left(x^{+}\right)
$$

with $r\left(x^{+}\right), a\left(x^{+}\right), b\left(x^{+}\right)$, functions of the affine parameter $x^{+}$defined through the null geodesics and $(\mathrm{J}, \mathrm{E})$-eigenvalue conditions

$$
-g_{t t} \dot{t}=1 \quad g_{\psi \psi} \dot{\psi}=\ell \quad g_{t t} \dot{t}^{2}+g_{\psi \psi} \dot{\psi}^{2}+g_{r r} \dot{r}^{2}=0
$$

Dots stand for the derivatives with respect to $x^{+}$. The first eq. in (2.4) fixes the normalization of the affine parameter by setting the energy $E=1$, while $\ell=J / E$ 
parametrizes the angular momentum. Eqs (2.4) are solved by ${ }^{1}$ :

$$
\dot{r}=\frac{\omega}{A B^{2} r} \quad \dot{a}=\frac{1}{A^{2}}-\frac{\ell^{2}}{B^{2} r^{2}} \quad \dot{b}=-\frac{\ell}{B^{2} r^{2}}
$$

with $A^{2} \equiv H^{\frac{p-7}{8}}, B^{2} \equiv H^{\frac{p+1}{8}}$ and $\omega\left(x^{+}\right) \equiv \sqrt{B^{2} r^{2}-\ell^{2} A^{2}}$. All functions in (2.5) carry an implicit dependence on $x^{+}$through $r\left(x^{+}\right)$.

In order to define the Penrose limit it is convenient to introduce the following change of coordinates:

$$
\begin{array}{ll}
\vec{x} & \rightarrow \Omega \mathbf{x} / A \quad \vec{y} \rightarrow \Omega \mathbf{y} /(B r) \quad r \rightarrow r\left(x^{+}\right) \quad \psi \rightarrow-b+\Omega z / \omega \\
t & \rightarrow-a-\ell \psi+\Omega^{2}\left[x^{-}+\frac{\partial_{+} \omega}{2 \omega} z^{2}+\frac{\partial_{+} A}{2 A} \mathbf{x}^{2}+\frac{\partial_{+}(B r \sin b)}{2 B r \sin b} \mathbf{y}^{2}\right]
\end{array}
$$

which reduces at $\Omega \rightarrow 0$ to the null geodesics (2.3). We parametrize by $\vec{y}$ the coordinates on the unitary sphere $S^{7-p}$. In the limit $\Omega \rightarrow 0$ they describe a point in $\mathbf{R}^{7-p}$ with coordinates $\mathbf{y}$. By plugging (2.6) into (2.1) and keeping only the leading terms in $\Omega \rightarrow 0$ one finds:

$$
\begin{aligned}
\Omega^{-2} d s^{2} & =2 d x^{+} d x^{-}-\left(m_{x}^{2} \mathbf{x}^{2}+m_{z}^{2} z^{2}+m_{y}^{2} \mathbf{y}^{2}\right) d x^{+2}+d z^{2}+d \mathbf{x}^{2}+d \mathbf{y}^{2} \\
\Omega^{-(p+1)} F_{p+2} & =2 \hat{f} d x^{+} \wedge d \operatorname{vol}\left(\mathbf{E}^{p}\right) \wedge d z
\end{aligned}
$$

with $e^{\phi-\phi_{\infty}}=\left(\frac{Q_{p}}{r^{7-p}}\right)^{\frac{3-p}{4}}$ and

$$
\begin{aligned}
& m_{x}^{2}=m_{z}^{2}=\frac{(7-p)^{2}}{256} Q_{p}^{-\frac{p+1}{4}} r^{-\frac{(p-3)^{2}}{4}}\left[\ell^{2}\left(31-2 p-p^{2}\right)+(p-3)^{2} Q_{p} r^{p-5}\right] \\
& m_{y}^{2}=\frac{(7-p)}{256} Q_{p}^{-\frac{p+1}{4}} r^{-\frac{(p-3)^{2}}{4}}\left[\ell^{2}\left(25+19 p-5 p^{2}+p^{3}\right)+(p-3)^{2}(7-p) Q_{p} r^{p-5}\right] \\
& 2 \hat{f}=(7-p) \ell Q^{\frac{-11+4 p-p^{2}}{16}} r^{\frac{(3-p)^{2}(5-p)}{16}}
\end{aligned}
$$

For $p=3$ one recovers the familiar pp-wave solution with masses $m_{x}^{2}=m_{y}^{2}=$ $m_{z}^{2}=\ell^{2} / Q_{p}$. On the other hand, for $p \neq 3$, the solution (2.7) involves non-trivial $x^{+}$-dependent dilaton, masses and RR-fluxes. A simple inspection of (2.5) reveals however that for $p \neq 5$ the radial evolution equation has a fixed point at the critical radius $[6]^{2}$

$$
r_{0}^{5-p} \approx \frac{Q_{p}}{\ell^{2}}
$$

where $\dot{r}=0$. Geodesics starting near this critical radius keeps close to it along $x^{+}$evolution leading to a pp-wave solution with constant masses, dilaton and RR-fluxes

\footnotetext{
${ }^{1}$ Solutions can be written in terms of hypergeometric functions, but the explicit form will not be needed here .

${ }^{2}$ The case $p=5$ should be treated independently and it will be omitted here (see [6, 12] for discussions on this case)
} 
[6]. Evaluating (2.8) at $r_{0}$ we are finally left with

$$
\begin{aligned}
m_{x}^{2} & =m_{z}^{2}=\frac{1}{32}(7-p)^{2}(5-p) m^{2} \\
m_{y}^{2} & =\frac{1}{32}(7-p)\left(11-4 p+p^{2}\right) m^{2} \\
e^{\frac{a_{p} \phi_{0}}{2}} 2 \hat{f} & =(7-p) m
\end{aligned}
$$

with $m^{2}=\ell^{\frac{29-10 p+p^{2}}{2(5-p)}} Q_{p}^{\frac{7-p}{2(p-5)}}$ and constant dilaton $e^{a_{p} \phi}=e^{a_{p} \phi_{0}}=\left(Q_{p} \ell^{p-7}\right)^{\frac{(3-p)^{2}}{4(5-p)}}$.

One can directly verify that (2.7) is indeed a solution of the ten dimensional Einstein equations. The only non-trivial component of the Einstein-Hilbert system:

$$
R_{++}=\frac{e^{a_{p} \stackrel{\phi}{\phi}}}{2 n !}\left(n F_{+A_{2} \ldots . . A_{n}} F_{+}^{A_{2} \ldots . . A_{n}}-\frac{n-1}{8} g_{++} F^{2}\right)
$$

requires

$$
\sum_{i} m_{i}^{2}=(p+1) m_{x}^{2}+(7-p) m_{y}^{2}=2 e^{a_{p} \phi_{0}} \hat{f}^{2}
$$

which is verified by $(2.10)^{3}$ Notice that the expressions (2.10) for the pp-wave masses differ substantially from those obtained in [6] using the string frame metric. This is not surprising since the two frames are related by a non-trivial $x^{+}$-dependent redefinition. We should stress that our choice here of working in the Einstein frame is not "ad hoc". Of course solutions coming from the Penrose limit (at the critical radius) using the string metric also fulfill the Einstein equations but due to the noncanonical kinetic term $e^{-2 \phi} \mathcal{R}$, a bit more care should be taken in discarding $\partial_{+}^{2} \phi$ terms.

For $p \neq 3$ the solutions (2.7) preserve half of the supersymmetries. Killing spinors equations can be solved in terms of spinor components $\epsilon$ satisfying $\Gamma^{+} \epsilon=0$ [3]. This matches the number of supersymmetries of the SYM theories living on the Dp-branes.

\section{KK supergravity harmonics}

In this section we compute the bosonic spectrum of type II,I supergravities in the F1/D1-wave geometry. We follow closely [13] where similar results were derived in the D3-wave case. The KK spectra found here should correspond to contractions of those in [8], where the type IIB/I linearized equations were solved in the warped $A d S_{3} \times S^{7}$ near horizon geometries. It would be nice to have a more concrete realization of this idea.

For definiteness we focus on the F1-pp wave case. $S L(2, \mathbb{Z})$ invariance of type IIB supergravity makes this vacuum indistinguishable from that of the D1-pp wave and therefore the two spectra are identical. States will be classified according to the

\footnotetext{
${ }^{3}$ In the following we will always reabsorve the $e^{\frac{a_{p} \phi_{0}}{2}}$ factor in the definition of $\hat{f}$ setting $e^{a_{p} \phi_{0}}=1$.
} 
$S O(6)$ R-symmetry group acting on the transverse $\mathbf{R}^{\mathbf{6}}$. This group is identified with the subgroup of the $S O(8) \mathcal{R}$-symmetry group in the two-dimensional gauge theory that survives the large R-charge BMN limit. In addition states will come in groups of 256 states at each level of the KK tower. This was also the case in the warped geometry [8] before taking the Penrose limit.

The spectrum of KK harmonics is determined by solving the linearized type IIB equations of motion in the pp-wave geometry. To linear order in the fluctuations around (2.7), type IIB supergravity fields can be written as (we borrow the notations of [14, 81):

$$
\begin{aligned}
g_{M N} & =\stackrel{\circ}{g}_{M N}+h_{M N} \\
P_{M} & =i \frac{\partial_{M} \tau}{2 \tau_{2}}=\frac{1}{2} \partial_{M} \Phi \\
q_{M} & =-\frac{\partial_{M} \tau_{1}}{2 \tau_{2}}=-\frac{e^{\phi_{0}}}{2} \partial_{M} C_{0} \\
G_{M N P} & =\stackrel{\circ}{G N P}_{M N}+\mathcal{G}_{M N P}=\frac{\varphi}{2} \stackrel{\circ}{M N P}_{M}+3 \partial_{[M} a_{N P]} \\
f_{M N P Q R} & =5 \partial_{[M} a_{N P Q R]}+\frac{5}{4}\left(a_{[M N}^{1} \partial_{P} a_{Q R]}^{2}-a_{[M N}^{2} \stackrel{\circ}{G}_{P Q R]}\right)
\end{aligned}
$$

where we have introduced the complex notation

$$
\begin{aligned}
\Phi & =\Phi_{1}+i \Phi_{2}=\varphi+i e^{\phi_{0}} \chi \\
a_{M N} & =a_{M N}^{1}+i a_{M N}^{2}=e^{\frac{\phi_{0}}{2}} a_{M N}^{N S}+i e^{-\frac{\phi_{0}}{2}} a_{M N}^{R}
\end{aligned}
$$

for fluctuations of the dilaton-axion and two-form systems respectively. In addition we will impose the light cone gauge conditions:

$$
h_{-M}=a_{-M}=a_{-M N P}=0
$$

to get rid of gauge degrees of freedom in the metric, two and four form. We will start by collecting the equations of motion for the various fields, which upon diagonalization reduce to a set of second order differential equations of the kind

$$
\left(\square+i \lambda_{s} \partial^{+}\right) \Phi_{s}=0
$$

with

$$
\square \equiv g^{M N} \partial_{M} \partial_{N}=2 \partial_{+} \partial_{-}+\partial_{I}^{2}-m_{I}^{2} y^{I 2} \partial_{-}^{2} .
$$

The index $s=1, . ., 256$ running over the 10D type IIB supergravity modes. We collect in $y^{I}=\{\mathbf{x}, z, \mathbf{y}\}, I=1, \ldots 8$, the transverse to the light-cone coordinates and denote by $m_{I}$ their associated masses. Identifying as in [13] our Hamiltonian by $H=-i \partial^{-}$, eq. (3.4) reduces to a system of eight harmonic oscillators with masses $m_{I}$ and energies

$$
E_{s}=\lambda_{s}-a+\sum_{I=1}^{8} m_{I} n_{I}
$$


with $n_{I}$ the oscillator occupation number and $a$ the zero point energy. $\lambda_{s}$ determine the shift in energy of the field $\Phi_{s}$ respect to other components at the same level in the KK-tower. The main task is to determine the spectrum of $\lambda$ 's by diagonalizing the linearized supergravity equations. Readers that are not interested in the details of this derivation can skip the next subsections directly to table 1, where the final results are summarized.

\subsection{Type IIB supergravity}

We start by considering the linearized equations of motion for type IIB supergravity around the F1-wave.

\section{Scalar Equation:}

The dilaton-axion equation of motion in the plane wave background reads:

$$
\stackrel{\circ}{D}^{M} P_{M}=\frac{1}{4} \stackrel{\circ}{G}^{-I J} \mathcal{G}_{-I J}
$$

In the light-cone gauge one has $\mathcal{G}_{-I J}=\partial^{+} a_{I J}$ and (3.7) can be rewritten as:

$$
\Phi-\frac{1}{2} c^{I J} \partial^{+} a_{I J}=0
$$

with $c_{I J}=\stackrel{\circ}{G}_{+I J}$ the three-form flux.

\section{Three-form Equation:}

The linearized equations for the complex three-form equations read:

$$
\delta D^{P} \stackrel{\circ}{G}_{M N P}+\stackrel{\circ}{D}^{P} \mathcal{G}_{M N P}=-P^{P} \stackrel{\circ}{G}_{M N P}^{*}-\frac{2}{3} i f_{M N P Q R} \stackrel{\circ}{G}^{M N P}
$$

As in 13 it is enough to solve for the components $(-I)$ and $(I J)$. The $(-I)$ component eqs:

$$
\stackrel{\circ}{D}^{P} \mathcal{G}_{-I P}=0 \quad \Rightarrow \partial^{P} a_{P I}=0
$$

can be used to rewrite

$$
\begin{aligned}
\stackrel{\circ}{D}^{P} \mathcal{G}_{I J P} & =\square a_{I J}+\frac{1}{2} c_{I J} \partial^{+} \varphi \\
\delta D^{P} \stackrel{\circ}{G}_{I J P} & =\frac{i}{2} c_{I J} \partial^{+} \Phi_{2}+2 \partial^{+} h_{K[I} c_{J] K}
\end{aligned}
$$

By plugging this in (3.9) and after some simple algebra one finds

$$
\square a_{I J}+\partial^{+}\left(c_{I J} \Phi+2 h_{K[I} c_{J] K}+2 i c^{K L} \hat{a}_{I J K L}\right)=0
$$

with $\hat{a}_{I_{1} . . I_{4}} \equiv a_{I_{1} . . I_{4}}+a_{\left[I_{1} I_{2}\right.}^{1} a_{\left.I_{3} I_{4}\right]}^{2}$.

\section{Metric Equations:}

The linearized graviton equations around the pp-wave geometry can be writen as

$$
r_{M N}=\frac{1}{8}\left(\stackrel{\circ}{G}_{M}^{P Q} \mathcal{G}_{N P Q}^{*}+\stackrel{\circ}{G}_{N}^{P Q} \mathcal{G}_{M P Q}^{*}-\frac{1}{6} \stackrel{\circ}{g}_{M N} \stackrel{\circ}{G}^{P Q R} \mathcal{G}_{P Q R}^{*}+\text { h.c. }\right)
$$


As in [13] the components $r_{--}=r_{-I}=0$ gives $h_{I I}=D^{m} h_{m I}=0$. This allows us to rewrite $r_{I J}=-\frac{1}{2} \square h_{I J}$ and (3.13) reads:

$$
\square h_{I J}+\frac{1}{2} \partial^{+}\left(c_{I K} a_{J K}^{*}+c_{J K} a_{I K}^{*}-\frac{1}{4} \delta_{I J} c_{K L} a_{K L}^{*}+\text { h.c. }\right)=0
$$

\section{Five-form equations}

Self-duality of the $\left(-I_{1} \ldots I_{4}\right)$ components gives

$$
\hat{a}_{I_{1} . . I_{4}}=\frac{1}{4 !} \epsilon_{I_{1} . . I_{4} J_{1} \ldots J_{4}} \hat{a}_{J_{1} . . J_{4}}
$$

Using the self-duality of the five-form and the definition (3.1) one writes

$$
\stackrel{\circ}{D}^{P} f_{P M_{1} . . M_{4}}=\frac{1}{23 !^{2}} \epsilon_{M_{1} \ldots M_{10}} F_{1}^{M_{5} M_{6} M_{7}} F_{2}^{M 8 M_{9} M_{10}}
$$

In particular for the $\left(-I_{1} . . I_{3}\right),\left(I_{1}, \ldots I_{4}\right)$ components one has:

$$
\begin{aligned}
\stackrel{\circ}{D}^{P} f_{P-I_{1} . . I_{3}} & =0 \Rightarrow \partial^{P} \hat{a}_{P I_{1} . . I_{3}}=0 \\
\stackrel{\circ}{D}^{P} f_{P I_{1} . . I_{4}} & =\frac{1}{8} \epsilon_{I_{1} . . I_{4} J_{1} . . J_{4}} F_{1}^{-J_{1} J_{2}} F_{2}^{+J_{3} J_{4}}
\end{aligned}
$$

Plugging in (3.16) one finally finds:

$$
\square \hat{a}_{I_{1} . . I_{4}}-\frac{1}{2} \partial^{+}\left[c_{\left[I_{1} I_{2}\right.} a_{\left.2 I_{3} I_{4}\right]}+\frac{1}{4 !} \epsilon_{I_{1} \ldots J_{4}} c^{\left[J_{1} J_{2}\right.} a_{2}^{\left.J_{3} J_{4}\right]}\right]=0
$$

\section{Spectrum of KK States:}

We now specify to a NSNS two-form flux $c_{12}=2 \hat{f}$ along the (12) plane. We split capital indices $I$ into $\alpha, \beta=1,2, i, j=1, \ldots 6$, according to whether they are parallel or perpendicular to the flux. Collecting equations (3.8), (3.12) (3.14) and (3.18), we find upon diagonalization a system of differential equations of the kind (3.4) with $\lambda$-eigenvalues displayed in table $\mathbb{1}$.

$\begin{array}{llll}\text { Fields } & S O(6) & \text { d.o.f. } & \lambda \\ h & \mathbf{1} & 1 & -4 \hat{f} \\ a_{\alpha i}^{N S}, \chi, a_{i j}^{R} & \mathbf{1}+2 \times \mathbf{6}+\mathbf{1 5} & 28 & -2 \hat{f} \\ h_{12}, \bar{h}_{\|}, h_{\|}, h_{i j}, a_{i j}^{N S}, a_{\alpha i}^{R}, a_{\alpha i_{1} . . i_{3}} & 3 \times \mathbf{1}+2 \times \mathbf{6}+\mathbf{1 5}+2 \times \mathbf{2 0} & 70 & 0 \\ \bar{a}_{\alpha i}^{N S}, \bar{\chi}, \bar{a}_{i j}^{R R} & \mathbf{1}+2 \times \mathbf{6}+\mathbf{1 5} & 28 & 2 \hat{f} \\ \bar{h} & \mathbf{1} & 1 & 4 \hat{f}\end{array}$

Table 1: KK bosonic spectrum of Type II supergravity.

We denote by $a_{\alpha i}, \chi, h, h_{\|}$some complex linear combinations of the axion/dilaton, metric and NSNS/RR forms. We display only the quantum numbers of the 128 bosonic states at the level 0 in the KK tower, i.e. in the bosonic zero mode ground state. States in the higher KK floors are still organize in groups of 256 states (128 bosons and 128 fermions). Energies are shifted by $n_{I} m_{I}$. SO(6)-representations of states in this higher KK levels can be found by tensoring the representations in the table with those of the harmonic oscillator zero modes $a_{0}^{I}$. 


\subsection{Type I supergravity}

The analysis above can be applied "mutatis mutandis" to the study of D1/F1-waves in type I/heterotic theory. The spectrum of KK states in the heterotic F1-wave can be read from that of type IIB by simply projecting out RR states and adding the contribution of $n_{V} \mathcal{N}=1$ vector multiplets. Similarly states in the type I D1-wave are related to those in the heterotic via S-duality.

The result of the $\Omega$-projection is displayed in table 2 .

$\begin{array}{llll}\text { Fields } & S O(6) & \text { d.o.f. } & \lambda \\ h & \mathbf{1} & 1 & -4 \hat{f} \\ a_{\alpha i} & 2 \times \mathbf{6} & 12 & -2 \hat{f} \\ h_{12}, \bar{h}_{\|}, h_{\|}, h_{i j}, a_{i j} & 3 \times \mathbf{1}+\mathbf{1 5}+\mathbf{2 0} & 38 & 0 \\ \bar{a}_{\alpha i} & 2 \times \mathbf{6} & 12 & 2 \hat{f} \\ \bar{h} & \mathbf{1} & 1 & 4 \hat{f}\end{array}$

Table 2: Type I Supergravity multiplet.

In addition we have $n_{V} \mathcal{N}=1$ vector multiplets with vector equations

$$
D_{M} F^{M N}-\frac{1}{2} \stackrel{\circ}{G P Q}_{N P} F^{P Q}=0,
$$

and $F_{M N} \equiv 2 e^{\frac{\delta}{2}} \partial_{[M} A_{N]}$. The equation for the (-)-component gives

$$
\stackrel{\circ}{D}^{P} F_{P-}=0 \quad \Rightarrow \partial^{P} a_{P}=0
$$

while for $I$ components we find

$$
\square a_{I}-c_{I J} \partial^{+} a_{J}=0
$$

Upon diagonalization, (3.21) reduces to our familiar differential equation with $\lambda$ eigenvalues given in table 3

As before, states in the higher levels of the KK tower can be found by acting with

$\begin{array}{llll}\text { Fields } & S O(6) & \text { d.o.f. } & \lambda \\ a & \mathbf{1} & 1 & -2 \hat{f} \\ a_{i} & \mathbf{6} & 6 & 0 \\ \bar{a} & \mathbf{1} & 1 & 2 \hat{f}\end{array}$

Table 3: Type I Gauge multiplet.

the oscillator bosonic zero modes $a_{0}^{I}$ and carry energies shifted by $n_{I} m_{I}$. Notice that now at each KK level, states are organized in groups of $128=64_{B}+64_{F}$ uncharged components and adjoint multiplets containing each $16=8_{B}+8_{F}$ physical degrees of freedom. 


\section{String theory on Dp-plane waves}

In this section we compute the helicity supertrace of string theory on Dp-waves (2.7) with masses $m_{I}$ and RR/NSNS fluxes $\hat{f}$. We follow closely [15], where similar techniques were applied in the six-dimensional context (see also [16] for related discussions). For definiteness we focus on type IIB. The general coupling of strings moving in a plane wave with NSNS/RR constant fluxes can be summarized in the following worldsheet Lagrangian:

$$
\mathcal{L}=\mathcal{L}_{0}+\mathcal{L}_{R}+\mathcal{L}_{N S}
$$

where

$$
\begin{aligned}
\mathcal{L}_{0} & =\frac{1}{2}\left(\partial_{+} Z^{i} \partial_{-} \bar{Z}^{i}-\mu_{i}^{2} Z^{i} \bar{Z}^{i}+\text { h.c. }\right)+S^{a} \partial_{+} S^{a}+\widetilde{S}^{a} \partial_{-} \widetilde{S}^{a} \\
\mathcal{L}_{R} & =-2 i f S^{a} \Pi_{a b} \widetilde{S}^{b} \\
\mathcal{L}_{N S} & =\frac{f_{i}}{2}\left(i \bar{Z}^{i} \partial_{+} Z^{i}-\bar{Z}^{i} \partial_{-} Z^{i}+\text { h.c. }\right)-\frac{i f_{i}}{2}\left(S \gamma^{i \bar{i}} S-\widetilde{S}^{a} \gamma_{a b}^{i \bar{i}} \widetilde{S}^{b}\right)
\end{aligned}
$$

We use the lightcone gauge $x^{+}=\alpha^{\prime} p^{+} \sigma_{0}$ and $\partial_{ \pm}=\partial_{0} \pm \partial_{1}$. Worldsheet masses are related to the spacetime variables via $\mu_{i}=2 p^{+} \alpha^{\prime} m_{i}, f=2 p^{+} \alpha^{\prime} \hat{f}, f_{i}=2 p^{+} \alpha^{\prime} H_{2 i 2 i-1+}$.

The Lagrangian densities $\mathcal{L}_{f}\left(\mathcal{L}_{v}\right)$ represent the coupling to RR(NSNS) fluxes while $\mathcal{L}_{0}$ describe a free string moving on a pp-wave. We have collected the eight real bosons into four complex ones and denote their masses by $\mu_{i}$. Sum over repeat indices $i, j=1, \ldots 4$ is implicitly understood. The RR fluxes are described by $f$ and couple to fermions through the mass term $\Pi=\gamma_{1} \ldots \gamma_{p+1}$.

NSNS fluxes are specified by $f_{i}$ 's. We have chosen $B_{+i}=\frac{i}{2} \hat{f}_{i} \bar{z}^{i}, B_{+\bar{i}}=-\frac{i}{2} \hat{f}_{i} z^{i}$ such that $H_{i \bar{i}+}=\hat{f}_{i}$. Notice that the coupling to fermions of NSNS fields are substantially different from that of RR-fields. Indeed the whole $\mathcal{L}_{N S}$-Lagrangian can be reabsorved by the worldsheet redefinition [15]

$$
Z^{i} \rightarrow e^{i f_{i} \sigma_{1}} Z^{i} \quad S \rightarrow e^{\frac{i}{2} f_{i} \sigma_{1} \gamma^{i \bar{i}}} S \quad \widetilde{S} \rightarrow e^{\frac{i}{2} \sigma_{1} f_{i} \gamma^{i \bar{i}}} \widetilde{S}
$$

and $\mu_{i}^{2} \rightarrow \mu_{i}^{2}-f_{i}^{2}$. The new fields satisfy twisted boundary conditions specified by $v_{i} \equiv a_{i} \tau+b_{i}=i f_{i} \tau_{2}$.

In the following we will restrict ourselves to RR-waves, but we will keep track of $\mathcal{R}$-charges by computing the one-loop partition functions in a given spin structure specified by $v_{i}=\bar{v}_{i}$. For future references we stress that the resulting helicity traces can be used, according to our discussion above, to extract one-loop correlation functions involving an arbitrary number of insertions of the NSNS B-fields on the RR plane wave.

We start by considering oriented closed string states. 


\subsection{The torus amplitude}

One-loop partition functions for type IIB on D3 pp-waves have been computed in [15, 17, 18]. In this section we compute helicity supertraces for string theories on Dp-plane waves. Helicity supertraces weight states according to their R-symmetry charges and allow us to perform a more refined comparison of multiplicities and charges with gauge/supergravity descriptions. In addition, as we have discussed earlier in this section, they provide a unifying description of D1/F1 plane wave string vacua.

An important new feature of string theory on general Dp-waves is that bosons and fermions carry in general different worldsheet masses $\mu_{i}, f$. The Lorentz $S O(8)$ symmetry is broken by pp-masses and RR-fluxes to its $S O(p+1) \times S O(7-p)$ subgroup. The helicity supertrace measures string multiplicities and charges under the $S O(2)^{4}$ Cartan subgroup of $S O(p+1) \times S O(7-p)$. More precisely, we define ${ }^{4}$

$$
Z\left(v_{i} \mid \tau, \bar{\tau}\right)=\operatorname{Tr}(-)^{F} e^{-2 \pi \tau_{2} H+2 \pi i \tau_{1} P+2 \pi v^{i} J_{i}}
$$

where

$$
\begin{aligned}
H & =\sum_{n \in \mathbb{Z}}\left(\omega_{n}^{i} N_{B n}^{i}+\omega_{n}^{f} N_{F n}\right)-a \quad a=2 \sum_{i=1}^{4}\left(\Delta_{f}-\Delta_{\mu_{i}}\right) \\
P & =\sum_{n \in \mathbb{Z}} n N_{n} \quad \omega_{n}^{i}=\sqrt{\mu_{i}^{2}+n^{2}}, \quad \omega_{n}^{f}=\sqrt{f^{2}+n^{2}} \\
v_{i} J^{i} & = \pm \sum_{n \in \mathbb{Z}}\left(v_{i} N_{B n}^{i}+\widetilde{v}_{i} N_{F n}^{i}\right) .
\end{aligned}
$$

We have built with $\mathbf{x}, z, \mathbf{y}$ four complex bosons and assigned them masses $\vec{\mu}$ according to (2.10). In addition we have introduced the quantities $\widetilde{v}_{i}$ defined through:

$$
\begin{aligned}
& \widetilde{v}_{1}=\frac{1}{2}\left(v_{1}+v_{2}+v_{3}+v_{4}\right) \\
& \widetilde{v}_{2}=\frac{1}{2}\left(v_{1}+v_{2}-v_{3}-v_{4}\right) \\
& \widetilde{v}_{3}=\frac{1}{2}\left(v_{1}-v_{2}-v_{3}+v_{4}\right) \\
& \widetilde{v}_{4}=\frac{1}{2}\left(v_{1}-v_{2}+v_{3}-v_{4}\right)
\end{aligned}
$$

which characterize the $S O(8)$ spinor representation under which worldsheet fermions transform.

The presence of the extra v-term twists the boundary conditions of worldsheet bosons and fermions by $v_{i}$ and $\widetilde{v}_{i}$ respectively. The twist is now along $\sigma_{0}$ in contrast with that in (4.3). The result follows with minor modifications from that in [17, 18]:

$$
Z(\vec{v} \mid \tau, \bar{\tau})=\prod_{i=1}^{4} \frac{\Theta_{0, \widetilde{v}_{i}}(f \mid \tau, \bar{\tau})}{\Theta_{0, v_{i}}\left(\mu_{i} \mid \tau, \bar{\tau}\right)}
$$

\footnotetext{
${ }^{4}$ We borrow the notations of 18 .
} 
An alternative derivation of this formula using the Lagrangian formalism is presented in the Appendix. At $v_{i}=0$, (4.7) reduces to the string partition function. Notice that unlike in the more familiar D3-brane case, bosonic and fermionic determinants do not cancel against each other even in the $\vec{v}=0$ case.

Focusing on the zero mode part of (4.7):

$$
\mathcal{Z}_{0}(\vec{v}, \tau)=\prod_{i=1}^{4} e^{2 \pi \tau_{2}\left(f-\mu_{i}\right)}\left|\frac{1-q^{f} e^{2 \pi i \tilde{v}_{i}}}{1-q^{\mu_{i}} e^{2 \pi i v_{i}}}\right|^{2}
$$

with $q=e^{2 \pi \tau}$, one easily recognizes the spectrum of Kaluza Klein supergravity states derived in the harmonic analysis above. The expansion of the numerator in powers of $q$ comprises 256 states out of which 128 are bosonic and 128 are fermionic states. More precisely besides a singlet state at level 0 , we find 8 states in the $\boldsymbol{8}_{s}$ spinor of $S O(8)$ at level one (term $q^{f}$ ), $28=\boldsymbol{8}_{s} \wedge \boldsymbol{8}_{s}$ states at level two (order $q^{2 f}$ ) and so on. Setting $p=1$, decomposing $S O(8)$ quantum numbers under $S O(6)$ and keeping only bosonic components, the spectrum in table 1 is reproduced. Different levels in the Kaluza-Klein tower are reached by expanding the denominator in (4.8). This corresponds to act with bosonic zero mode creation operators. Theses terms explicitly break the $S O(8)$ symmetry down to $S O(6)$. Degeneracies of these states are in agreement with that of the set of harmonic oscillators with masses $\mu_{i}$ found in the supergravity description.

It is also not hard to associate to each state in (4.7) a BMN operator of large $\mathcal{R}$-charge in the dual $p+1$-dimensional gauge theory. The dictionary follows straight from that in [2] by simply reorganizing the spectrum of operators respect to $S O(p+$ 1) $\times S O(7-p)$ rather than $S O(4) \times S O(4)$. The string vacuum is again identified with the $\frac{1}{\sqrt{J} N^{J}} \operatorname{Tr} Z^{J}$ operator. The 256 operators associated with states at a given "level" in the KK tower are related by actions of the eight fermion gaugino components $\chi_{J=\frac{1}{2}}$ in the $d=p+1$-dimensional theory. The main difference with the $p=3$ case, is that insertions in the traces of operators $D_{\alpha} Z$ and $\phi^{i}(\alpha=1, . . p+1, i=1, . .7-p)$ carry different energies due to the difference in the longitudinal and transverse masses. This is related to the fact that the radii of the original $A d S_{p+2}$ and $S^{8-p}$ are different. It would be nice to explain this rich structure of the energy spectrum in the gauge theory side.

Before leaving this section it is worth to spend some words in the flat space limit of our formulas. For this purpose it is useful to come back to the derivation of the helicity trace presented in the Appendix. As it has been recognized in various places in the literature (see [11, 20] for example), the massless limit is far from smooth due to non-standard bosonic zero mode contributions implicitly taken into account in (4.7). More precisely, for $\mu=0$ the computation of the bosonic determinant (5.2) in the Appendix, should be replaced by that of $\operatorname{det}^{\prime} \square_{v}$ where the $n_{1}=n_{2}=0$ constant mode is omitted. The integration over this constant mode $x_{0}$ gives the regularized 
volume of the target space. A sensible comparison with flat space results is better performed in terms of the primed partition function

$$
Z^{\prime}(\vec{v} \mid \tau, \bar{\tau}) \equiv Z(\vec{v} \mid \tau, \bar{\tau}) \mathcal{V}_{0}\left(\tau_{2}\right)=Z(\vec{v} \mid \tau, \bar{\tau}) \tau_{2}^{-4} \prod_{i=1}^{4}\left|v_{i}+i \mu_{i} \tau_{2}\right|^{2}
$$

where contributions coming from the constant mode are omitted and collected in $\mathcal{V}_{0}^{-1}\left(\tau_{2}\right)$. Notice that both $Z$ and $Z^{\prime}$ are modular invariant since $\mathcal{V}_{0}\left(\tau_{2}\right)$ is invariant by itself under the modular transformation (5.7). In particular for $\vec{v}=0$ the two definitions differ by powers of the modular invariant combination $\mu_{i}^{2} \tau_{2}{ }^{5}$. In terms of (4.9) the torus amplitude can be written as

$$
\mathcal{T}=\int \frac{d^{2} \tau}{\tau_{2}^{2}} d p^{+} d p^{-} e^{-2 \pi i \alpha^{\prime} p^{+} p^{-} \tau_{2}} \mathcal{V}_{0}\left(\tau_{2}\right) Z^{\prime}(\vec{v} \mid \tau, \bar{\tau})
$$

The flat space limit is recovered by sending $\mu \rightarrow 0$ :

$$
\lim _{\mu \rightarrow 0} Z^{\prime}(\vec{v} \mid \tau, \bar{\tau})=\frac{1}{\tau_{2}^{4}} \prod_{i=1}^{4}\left|v_{i} \frac{\vartheta_{1}\left(\widetilde{v}_{i}\right)}{\vartheta_{1}\left(v_{i}\right)}\right|^{2}
$$

and replacing $\mathcal{V}_{0}\left(\tau_{2}\right)$ by the regularized volume $V_{0}$ of the target space. According to [19, 20] integration over $p^{ \pm}$set $p^{+}$(and therefore $\mu, f$ ) to zero and therefore the vacuum energy vanishes at $\vec{v}=0$ as expected.

\subsection{Unoriented and open strings}

In this section we consider open descendants of type IIB superstrings on D1-waves. Open descendants of type IIB on D3-waves were first studied in [21]. As in that case the open string descendant here will be constructed by adding Dp-Op worldsheet boundaries to the D1-wave type IIB vacuum. We follow standard open string descendant techniques (for a review and a complete list of references see [22].) Quantization of open strings moving on RR-plane waves have been studied in various contexts [9, 10, 11, 23]. The selection rules for the allowed Dp-Op systems in a D1-wave geometry follow from those been derived in [9, 10, 11. It is convenient to introduce the Dp-mass operator $M \equiv \Pi \Pi_{p}$, where $\Pi=\gamma^{12}$ and $\Pi_{p}=\gamma^{12 . . p-1}$ are the chirality operators along the D1-flux plane and $\mathrm{Dp} / \mathrm{Op}$ plane respectively. A consistent $\mathrm{Dp} / \mathrm{Op}$ system is defined roughly by requiring that $\operatorname{Tr} M=0$ and $M^{2}=-1^{6}$. This implies that the difference between the number of NN and DD directions is $2 \bmod 4$. These conditions are clearly satisfied by Dp-Op systems with $\mathrm{p}=5,9$ oriented along

\footnotetext{
${ }^{5}$ The origin of this normalization factor can be traced in the ratio between the partition function of a $2 \mathrm{~d}$ harmonic oscillator $\frac{1}{\mu^{2} \tau_{2}^{2}}=\int d \mathbf{x}_{0} d \mathbf{p} e^{-\pi \tau_{2}\left(\mathbf{p}^{2}+\mu^{2} \mathbf{x}_{0}^{2}\right)}$ and its free particle limit $\frac{V}{\tau_{2}}=\int d \mathbf{x}_{0} d \mathbf{p} e^{-\pi \tau_{2} \mathbf{p}^{2}}$.

${ }^{6}$ These two conditions ensure that the effective zero mode Hamiltonian $H \sim S_{0}^{a} M_{a b} S_{0}^{b}$ reproduces the right fermionic contributions in the closed string channel [1]
} 
$x^{ \pm}$and parallel to the D1-plane flux. We will focus on these two cases, although our formulas are derived in a rather more general perspective. These systems are of particular interest since they should presumably provide S-dual string duals of heterotic string on F1-plane waves.

Open string amplitudes and tadpole conditions for self-dual pp-wave vacua have been worked out recently in [19, 20]. The extension of these results to our case is straightforward. Apart from the zero mode part the Klein and open string amplitudes are roughly the square root of (4.7):

$$
\begin{aligned}
\mathcal{K} & =\int \frac{d^{2} \tau}{\tau_{2}^{2}} d p^{+} d p^{-} e^{-2 \pi \alpha^{\prime} p^{+} p^{-} \tau_{2}} \frac{Z^{\frac{1}{2}}\left(2 i \tau_{2}\right)}{Z_{0}^{\frac{1}{2}}\left(2 i \tau_{2}\right)} \mathcal{K}_{0} \\
\mathcal{A} & =N^{2} \int \frac{d^{2} \tau}{\tau_{2}^{2}} d p^{+} d p^{-} e^{-2 \pi \alpha^{\prime} p^{+} p^{-} \tau_{2}} \frac{Z^{\frac{1}{2}}\left(\frac{i \tau_{2}}{2}\right)}{Z_{0}^{\frac{1}{2}}\left(\frac{i \tau_{2}}{2}\right)} \mathcal{A}_{0} \\
\mathcal{M} & = \pm N \int \frac{d^{2} \tau}{\tau_{2}^{2}} d p^{+} d p^{-} e^{-2 \pi \alpha^{\prime} p^{+} p^{-} \tau_{2}} \frac{Z^{\frac{1}{2}}\left(\frac{i \tau_{2}}{2}+\frac{1}{2}\right)}{Z_{0}^{\frac{1}{2}}\left(\frac{i \tau_{2}}{2}+\frac{1}{2}\right)} \mathcal{M}_{0}
\end{aligned}
$$

In (4.12) and throughout this section we have settled $\vec{v}=0$. By $N$ we denote the number of branes, while \pm denote the possible orthogonal or symplectic choice of Moebius projections in the open string sector. Finally we have denoted by $\mathcal{Z}_{0}, \mathcal{K}_{0}$, $\mathcal{A}_{0} \mathcal{M}_{0}$ the zero mode contributions to the torus, Klein, Annulus and Moebius strip amplitudes respectively:

$$
\begin{aligned}
\mathcal{Z}_{0}\left(i \tau_{2}\right) & =\prod_{i=1}^{4} q^{\left(\mu_{i}-f\right)} \frac{\left(1-q^{f}\right)^{2}}{\left(1-q^{\mu_{i}}\right)^{2}} \\
\mathcal{K}_{0} & =\prod_{i=1}^{4} q^{\left(\mu_{i}-f\right)} \frac{\left(1-q^{2 f}\right)}{\left(1+\epsilon_{i} q^{\mu_{i}}\right)^{2}} \\
\mathcal{A}_{0}=\mathcal{M}_{0} & =\prod_{i=1}^{4} q^{\frac{1}{4}\left(\epsilon_{i}+1\right) \mu_{i}-\frac{1}{4} f} \frac{\left(1-q^{\frac{f}{2}}\right)}{\left(1-q^{\frac{\mu_{i}}{2}}\right)^{\epsilon_{i}+1}}
\end{aligned}
$$

Here $q=e^{-2 \pi \tau_{2}}$ and $\epsilon_{i}=+,-$ for NN and DD directions respectively, i.e.

$$
\begin{array}{ll}
\mathrm{D} 9 / \mathrm{O} 9 & \epsilon_{1}=\epsilon_{2}=\epsilon_{3}=\epsilon_{4}=+1 \\
\mathrm{D} 5 / \mathrm{O} 5 & \epsilon_{1}=\epsilon_{2}=+1 \quad \epsilon_{3}=\epsilon_{4}=-1
\end{array}
$$

The $\epsilon_{i}$ in the Klein bottle amplitude reflects the minus eigenvalue of the $\Omega Z_{2^{-}}$ projection acting on DD-bosons. The action on fermions can instead be read off from the eigenvalues under $\Pi_{p}=\gamma^{1} \ldots \gamma^{p-1}$, i.e. half plus and half minus. Open string zero mode contributions come on the other hand from bosonic zero modes $a_{0}^{I_{N}}$ along NN directions and fermions with a definite $\Pi_{p}$-chirality. 
The amplitudes (4.12) define (by construction) a projection of the closed and open string spectrum defined by the oriented torus and annulus amplitudes. However in order to state the consistency of the string vacuum one should ensure that worldsheet boundaries couple consistently to the bulk modes, i.e. that the sum of the three amplitudes (4.12) in the closed string channel reconstruct a complete square $(|B\rangle+|C\rangle)^{2}$. Using the modular invariance of $Z(\tau)$ the amplitudes (4.12) can be rewritten in the closed string channel as

$$
\begin{aligned}
\mathcal{K} & =\frac{1}{2} \int d \ell d \widetilde{p}^{+} d \widetilde{p}^{-} e^{-\pi \alpha^{\prime} \widetilde{p}^{+} \widetilde{p}^{-}} Z^{\frac{1}{2}}(i \ell) \widetilde{\mathcal{K}}_{0} \\
\mathcal{A} & =\frac{1}{8} \int d \ell d \widetilde{p}^{+} d \widetilde{p}^{-} e^{-\pi \alpha^{\prime} \widetilde{p}^{+} \widetilde{p}^{-}} Z^{\frac{1}{2}}(i \ell) \widetilde{\mathcal{A}}_{0} \\
\mathcal{M} & =\frac{1}{2} \int d \ell d \widetilde{p}^{+} d \widetilde{p}^{-} e^{-\pi \alpha^{\prime} \widetilde{p}^{+} \widetilde{p}^{-}} Z^{\frac{1}{2}}\left(i \ell+\frac{1}{2}\right) \widetilde{\mathcal{M}}_{0}
\end{aligned}
$$

By $\ell$ we have denoted the uniforming length, given in terms of the original variables in (4.12) via $\ell_{K}=\frac{1}{2 \tau_{2}}, \ell_{A}=\frac{2}{\tau_{2}}, \ell_{M}=\frac{1}{2 \tau_{2}}$. Although it is not explicitly indicated, amplitudes in the transverse channel are evaluated on masses $\widetilde{\mu}, \widetilde{f}$ related to those in the direct channel by $S$ or $S T^{2} S T$ modular transformations. More precisely $\widetilde{\mu}, \widetilde{f}=$ $\frac{\mu}{\ell}, \frac{f}{\ell}$ for the Klein and Annulus amplitude and $\widetilde{\mu}, \widetilde{f}=\frac{\mu}{2 \ell}, \frac{f}{2 \ell}$ for the Moebius. Finally $\widetilde{p}^{+} \widetilde{p}^{-}=\frac{p^{+} p^{-}}{\ell}$ for the Klein and Moebius amplitude while $\widetilde{p}^{+} \widetilde{p}^{-}=\frac{4 p^{+} p^{-}}{\ell}$ for the annulus. Rewriting (4.15) in terms of the transverse variables we find:

$$
\begin{aligned}
\widetilde{\mathcal{K}}_{0} & \equiv \frac{\mathcal{K}_{0}}{Z_{0}^{\frac{1}{2}}\left(2 i \tau_{2}\right)}=\prod_{i=1}^{4}\left(\frac{1+e^{-\pi \widetilde{\mu}_{i}}}{1-e^{-\pi \widetilde{\mu}_{i}}}\right)^{\epsilon_{i}} \\
\widetilde{\mathcal{A}}_{0} & \equiv \frac{\mathcal{A}_{0}}{Z_{0}^{\frac{1}{2}}\left(\frac{i \tau_{2}}{2}\right)}=\prod_{i=1}^{4} e^{-\pi \widetilde{\mu}_{i}}\left(1-e^{-2 \pi \widetilde{\mu}_{i}}\right)^{-\epsilon_{i}} \\
\widetilde{\mathcal{M}}_{0} & \equiv \frac{\mathcal{M}_{0}}{Z_{0}^{\frac{1}{2}}\left(\frac{i \tau_{2}}{2}+\frac{1}{2}\right)}=\prod_{i=1}^{4} e^{-\frac{1}{2} \pi \widetilde{\mu}_{i}}\left(1-e^{-\pi \widetilde{\mu}_{i}}\right)^{-\epsilon_{i}}
\end{aligned}
$$

Remarkably after summing up $\frac{1}{2} \mathcal{K}_{0}+\frac{N^{2}}{8} \mathcal{A}_{0} \pm \frac{N}{2} \mathcal{M}_{0}$ a complete square $(|B\rangle+|C\rangle)^{2}$ is reconstructed

$$
\frac{1}{2}\left[1 \pm \frac{N}{2} \prod_{i=1}^{4} e^{-\frac{1}{2} \pi \widetilde{\mu}_{i}}\left(1+e^{-\pi \widetilde{\mu}_{i}}\right)^{-\epsilon_{i}}\right]^{2} \prod_{i=1}^{4}\left(\frac{1+e^{-\pi \widetilde{\mu}_{i}}}{1-e^{-\pi \widetilde{\mu}_{i}}}\right)^{\epsilon_{i}}
$$

For the D7-O7 system in the D3-wave $\mu_{i}=f=\mu$ one recovers the results of [20]. It would be nice to give an interpretation of the two terms in (4.17) in terms of reflection coefficients of closed string states in front of the boundaries/crosscaps. Finally following [19, 20], we can derive tadpole conditions ${ }^{7}$ by requiring that the

\footnotetext{
${ }^{7}$ Notice that amplitudes (4.12) are in the odd-spin structure are therefore the tadpole 4.17) can be related to anomalies according to [24].
} 
combination inside the square in (4.17) cancels in the $\mu \rightarrow 0$ limit. This boils down to choose the orthogonal projection for the Moebius and

$$
N=2^{p-4}
$$

which matches the familiar result in flat space.

In particular specifying to $p=9$ we conclude that 32 D9-branes are need to cancel the $\mathrm{O} 9$ tadpole in the D1-wave. Similarly the charge of a O5-plane is canceled by 2 D5-branes sited at the $R^{4} / Z_{2}$ singularity.

\section{Summary and open questions}

In this paper we applied exact CFT techniques to solvable string models on RR/NSNS plane wave geometries. Dp-waves descend from Penrose limits of non-conformal Dp-brane geometries along critical null geodesics. The solutions preserve sixteen supercharges and provide the simplest examples of string vacua in the presence of non-self dual RR fluxes. We have focused on D1-plane waves of typeIIB/type I theory. These systems are natural candidates to describe large $\mathcal{R}$-charge sectors of the type IIA/heterotic matrix string theories [25]. Before the Penrose limit is taken they are described by warped $A d S_{3} \times S^{7}$ geometries and have been recently studied in [8]. Here we derive the bosonic KK spectrum of $\mathcal{N}=1,2$ supergravity on D1/F1 plane waves. KK particles organize nicely in groups of 256 (or 128 in type I case) states at each floor of the KK tower. An important difference with the case of self-dual plane waves is that excitations along or perpendicular to the RR-flux carry different energies. This is due to the fact that the radii of the $A d S_{p+2}$ and $S^{8-p}$ spaces in the parent warped geometry are different.

In section 4 , we computed helicity traces counting multiplicities and charges of string states in the pp-wave background. It would be nice to apply these helicity formulas to the study of correlation functions in pp-wave backgrounds. As discussed earlier in that section, the helicity trace provides an efficient description of the coupling of RR-plane waves to the NSNS B-field. By expanding the resulting modular functions one can derive one-loop correlation functions involving an arbitrary number of $H=d B$ insertions. This can be used to test the gauge/pp-wave correspondence beyond the planar level.

Finally in subsection 4.2 we considered open descendants built from D1-wave type IIB vacuum by adding Dp-Op branes with $p=5,9$. Klein, Annulus and Moebius amplitudes are computed. We test the consistency of the open string descendant by showing that after modular transformations to the closed string channel the three amplitudes combine themselves into a complete square. Op-tadpoles are shown to be canceled by the introduction $2^{p-4}$ Dp-planes much in the same way as in flat space time. 
Our results show that Dp-waves (2.7) share most of the nice integrability properties of the more familiar pp-waves supported by self-dual fluxes. Besides the applications to holography the study of these string vacua is interesting by itself since they provide the simplest string solution with non-self-dual RR fluxes and constant dilaton. D1-waves of type IIB/I are related via S-duality to pure NSNS F1-plane waves in type IIB/heterotic ten dimensional string theories. Although RR-fluxes are present, the two sides of the duality is described by a solvable string theory. String dualities in the presence of RR-fluxes are fully unexplored. The D1/F1-wave string dual pairs under consideration here can provide a handle for this physics.

Finally according to [27], self-dual D1-waves are related to blowing up deformations of the dual $M^{N} / S_{N}$ SCFT's describing the infrared regime of D1D5 systems on $M=T^{4}, K 3$. It would be nice to understand whether these results extend to the 10D D1-waves studied here. The candidate SCFT's are in this case given in terms of second quantized type IIB/heterotic strings on $\left(R^{8}\right)^{N} / S_{N}$ [25].

\section{Acknowledgements}

We wish to thank A.B. Hammou, R. Russo, H. Samtleben, M. Taylor, M. Trigiante and S. Vandoren for useful discussions. This work is partly supported by EU contract HPRN-CT-2000-00122.

\section{Appendix: Partition functions and generalized modular forms}

In this appendix we compute, using the Lagrangian formalism, the one-loop partition function of two dimensional massive fields coupled to a $U(1)$ current. We adopt the $\xi$-regularization formalism [26].

Let us start by considering a single complex boson with two-dimensional action

$$
\mathcal{L}=\partial Z \bar{\partial} \bar{Z}-\frac{\pi \bar{v}}{\tau_{2}} \bar{Z} \partial Z-\frac{\pi v}{\tau_{2}} \bar{Z} \bar{\partial} Z+\pi^{2}\left(\mu^{2}+\frac{v \bar{v}}{\tau_{2}^{2}}\right) \bar{Z} Z+\text { h.c. }
$$

The coupling of the boson to the $U(1)$ current is described by the complex number $v$, that will be written as $v=a \tau+b$. After expanding in Fourier modes

$$
Z=\sum_{n_{1}, n_{2}} c_{n_{1}, n_{2}} e^{\frac{\pi}{\tau_{2}}\left[n_{2}(z-\bar{z})+n_{1}(\tau \bar{z}-\bar{\tau} z)\right]}
$$

and performing the Gaussian integrals, it results in the inverse of the determinant:

$$
\begin{aligned}
\operatorname{det} \square_{v} & =\prod_{\left(n_{1}, n_{2}\right)} \frac{\pi^{2}}{\tau_{2}^{2}}\left(\left|n_{2}-n_{1} \tau-v\right|^{2}+\mu^{2} \tau_{2}^{2}\right) \\
& =\prod_{\left(n_{1}, n_{2}\right)} \frac{\pi^{2}}{\tau_{2}^{2}}\left|n_{2}-\left(n_{1}+a\right) \tau_{1}-b+i \tau_{2} \sqrt{\left(n_{1}+a\right)^{2}+\mu^{2}}\right|^{2}
\end{aligned}
$$


Performing the $n_{2}$ infinite product by means of the $\xi$-regularization formulas

$$
\prod_{n=-\infty}^{\infty}(n-a)=2 i \sin \pi a \quad \prod_{n=-\infty}^{\infty} a=1
$$

we are finally left with

$$
\operatorname{det} \square_{v}=\Theta_{a, b}(\mu \mid \tau, \bar{\tau}) \equiv e^{4 \pi \tau_{2} \Delta_{\mu}} \prod_{\left(n_{1} \in \mathbb{Z}+a\right)}\left|1-e^{-2 \pi \tau_{2} \sqrt{\mu^{2}+n_{1}^{2}}+2 \pi i n_{1} \tau_{1}+2 \pi i b}\right|^{2}
$$

where $\Delta_{\mu} \equiv \frac{1}{2} \sum_{n=-\infty}^{\infty} \sqrt{\mu^{2}+n^{2}}$. In the massless limit $\mu \rightarrow 0$ the generalized function (5.5) reduce to the familiar theta function:

$$
\lim _{\mu \rightarrow 0} \Theta_{a, b}(\mu \mid \tau, \bar{\tau})=e^{-2 \pi \tau_{2} a^{2}}\left|\frac{\vartheta_{1}(a \tau+b \mid \tau)}{\eta}\right|^{2}
$$

The modular invariance of this form can be read from (5.3), which is clearly invariant under ${ }^{8}$ :

$$
\tau \rightarrow-\frac{1}{\tau} \quad \mu \rightarrow \mu|\tau| \quad v \rightarrow-\frac{v}{\tau} \quad n_{1} \leftrightarrow n_{2} .
$$

According to $v^{\prime}=a^{\prime} \tau^{\prime}+b^{\prime}=-\frac{v}{\tau}$, we see that spin structures transform under the modular group as $a^{\prime}=b, b^{\prime}=-a$.

Similar manipulations hold for the fermionic determinants with the result (5.5) now coming in the numerator.

\section{References}

[1] J. M. Maldacena, Adv. Theor. Math. Phys. 2 (1998) 231; Int. J. Theor. Phys. 38 (1999) 1113, hep-th/9711200.

[2] D. Berenstein, J. M. Maldacena and H. Nastase, JHEP 0204 (2002) 013, hep-th/0202021.

[3] M. Blau, J. Figueroa-O'Farrill, C. Hull and G. Papadopoulos, JHEP 0201 (2002) 047 ,hep-th/0110242 . M. Blau, J. Figueroa-O'Farrill, C. Hull and G. Papadopoulos, Class. Quant. Grav. 19 (2002) L87 ,hep-th/0201081 . M. Blau, J. Figueroa-O'Farrill and G. Papadopoulos, Class. Quant. Grav. 19 (2002) 4753 ,hep-th/0202111 .

[4] A. A. Tseytlin, Class. Quant. Grav. 12 (1995) 2365 ,hep-th/9505052 .

[5] E. G. Gimon, L. A. Pando Zayas and J. Sonnenschein, Penrose limits and RG flows, hep-th/0206033.

[6] H. Fuji, K. Ito and Y. Sekino, "Penrose limit and string theories on various brane backgrounds", hep-th/0209004.

\footnotetext{
${ }^{8} \mathrm{In}$ proving this one uses $\prod_{n=-\infty}^{\infty} \tau_{2}=1$.
} 
[7] N. Itzhaki, J. M. Maldacena, J. Sonnenschein and S. Yankielowicz, Phys. Rev. D 58 (1998) 046004, hep-th/9802042. H. J. Boonstra, K. Skenderis and P. K. Townsend, JHEP 9901 (1999) 003, hep-th/9807137. K. Behrndt, E. Bergshoeff, R. Halbersma and J. P. van der Schaar, Class. Quant. Grav. 16 (1999) 3517, hep-th/9907006.

T. Gherghetta and Y. Oz, Phys. Rev. D 65 (2002) 046001, hep-th/0106255.

[8] J. F. Morales and H. Samtleben, JHEP 0208 (2002) 042 ,hep-th/0206247 .

[9] M. Billo and I. Pesando, Phys. Lett. B 536 (2002) 121 ,hep-th/0203028 .

[10] A. Dabholkar and S. Parvizi, Nucl. Phys. B 641 (2002) 223 ,hep-th/0203231 .

[11] O. Bergman, M. R. Gaberdiel and M. B. Green, "D-brane interactions in type IIB plane-wave background,", hep-th/0205183.

[12] Y. Oz and T. Sakai, Phys. Lett. B 544 (2002) 321 ,hep-th/0207223 .

[13] R. R. Metsaev and A. A. Tseytlin, Phys. Rev. D 65 (2002) 126004 , hep-th/0202109.

[14] J. H. Schwarz, Nucl. Phys. B 226 (1983) 269.

[15] J. G. Russo and A. A. Tseytlin, JHEP 0204 (2002) 021 ,hep-th/0202179 .

[16] E. Kiritsis and B. Pioline, JHEP 0208 (2002) 048 ,hep-th/0204004 . S. Mizoguchi, T. Mogami and Y. Satoh, "Penrose limits and Green-Schwarz strings", hep-th/0209043.

[17] T. Takayanagi, "Modular invariance of strings on pp-waves with RR-flux", hep-th/0206010.

[18] Y. Sugawara, "Thermal amplitudes in DLCQ superstrings on pp-waves", hep-th/0209145.

[19] A. Sinha and N. V. Suryanarayana, "Tadpole analysis of orientifolded plane-waves", hep-th/0209247.

[20] A. B. Hammou, "One loop partition function in plane waves $R$ - $R$ background", hep-th/0209265.

[21] D. Berenstein, E. Gava, J. M. Maldacena, K. S. Narain and H. Nastase, "Open strings on plane waves and their Yang-Mills duals", hep-th/0203249.

[22] C. Angelantonj and A. Sagnotti, "Open strings", hep-th/0204089.

[23] P. Lee and J. w. Park, "Open strings in PP-wave background from defect conformal field theory", hep-th/0203257. A. Kumar, R. R. Nayak and Sanjay, Phys. Lett. B 541 (2002) 183, hep-th/0204025. K. Skenderis and M. Taylor, JHEP 0206 (2002) 025, hep-th/0204054. H. Takayanagi and T. Takayanagi, JHEP 0205 (2002) 012 ,hep-th/0204234 . M. Alishahiha and A. Kumar, Phys. Lett. B 542 (2002) 130, hep-th/0205134 . S. G. Naculich, H. J. Schnitzer and N. Wyllard, "pp-wave limits 
and orientifolds", hep-th/0206094. M. Alishahiha, M. A. Ganjali, A. Ghodsi and S. Parvizi, "On type IIA string theory on the PP-wave background,", hep-th/0207037. K. Sugiyama and K. Yoshida, "Type IIA string and matrix string on pp-wave,", hep-th/0208029. S. j. Hyun and H. j. Shin, "Solvable $N=(4,4)$ type IIa string theory in plane-wave background and D-branes", hep-th/0210158.

[24] M. Bianchi and J. F. Morales, JHEP 0003 (2000) 030, hep-th/0002149; hep-th/0101104.

[25] R. Dijkgraaf, E. Verlinde and H. Verlinde, Nucl. Phys. B 500 (1997), hep-th/9703030; Nucl. Phys. Proc. Suppl. 68 (1998) 28. T. Banks and L. Motl, JHEP 9712 (1997) 004, hep-th/9703218; D. A. Lowe, Phys. Lett. B 403 (1997) 243, hep-th/9704041; S. J. Rey, Nucl. Phys. B 502 (1997) 170, hep-th/9704158. E. Gava, J. F. Morales, K. S. Narain and G. Thompson, Nucl. Phys. B 528 (1998) 95, hep-th/9801128.

[26] P. Ginsparg, HUTP/89/A027 Lectures given at Trieste Spring School, Trieste, Italy, Apr 3-14, 1989.

[27] E. Gava and K. S. Narain, "Proving the pp-wave / CFT(2) duality", hep-th/0208081. 